\title{
EL JUEGO EN LA EDUCACIÓN FÍSICA ESCOLAR URUGUAYA: SENTIDOS Y LEGITIMACIONES DESDE LO CURRICULAR
}

The Game in Uruguayan school physical education: goals and legitimations from the perspective of the curricular proposal

O jogo na educação fisica escolar uruguaia: sentidos e legitimações do currículo

\section{Pablo Zinola}

Universidad de la República de Uruguay. Instituto Superior de Educación, Montevideo, Uruguay. Telf.: +598220296. Correo electrónico:

\section{pazinola@gmail.com}

\section{Cecilia Ruegger}

Universidad de la República de Uruguay. Instituto Superior de Educación, Montevideo, Uruguay. Telf.: +59899290160. Correo electrónico: ceciliaruegger@gmail.com

\section{Ana Torrón}

Universidad de la República de Uruguay. Instituto Superior de Educación, Montevideo, Uruguay. Telf.: +59899163550. Correo electrónico: anitentorron@gmail.com

\section{Resumen}

El juego es uno de los contenidos fundantes de la educación física escolar uruguaya. Su inclusión como contenido a ser enseñado en la escuela data del primer programa de educación física escolar (1911). Desde esa inclusión temprana, pueden rastrearse múltiples discursos legitimadores. En diferentes épocas se fueron estableciendo diversas representaciones, imaginarios y prácticas en relación al juego. Nos proponemos visualizar los discursos actuales que dan sentido a este tradicional contenido. Optamos por focalizar la atención en el programa escolar vigente, entendiendo que este documento nos ofrece una posibilidad para rastrear aquello que es definido y validado en la actualidad para la institución escolar. En este texto analizamos los lineamientos que se definen en el actual Programa del Área del Conocimiento Corporal (ANEP, 2008). Se trata de un documento 
programático que pretende "una nueva mirada al valor del juego". Como punto de partida, tomamos como referencia los tres ejes que presenta el programa en torno al juego, es decir: concepto, metodología y contenido. En esta articulación de categorías, el programa da cuenta de diversos sentidos y legitimaciones, evidenciando algunas tensiones. La complejidad central de esta indagación refiere a un doble problema para la educación física: el juego como elemento pedagógico y el juego como objeto de enseñanza. Es importante señalar que algunos de los problemas identificados en este análisis, no son exclusivos de este programa, sino que reflejan una realidad siempre conflictiva del juego en el ámbito educativo en general y en la educación física en particular.

Palabras clave: juego, educación física, programa escolar.

\begin{abstract}
The game is one of the founding contents in Uruguayan school physical education. Its inclusion within the subjects to be taught in school dates back to the first school physical education program (1911). From that early inclusion, numerous legitimating discourses can be traced. At different times, diverse representations, imaginaries and practices were established in relation to the game. Therefore, it is the aim of our research to analyze the current discourses which assign meaning to this traditional subject matter. We decided to focus our attention on the present school curricula, as we understand it offers us the possibility to track down what is being currently defined and validated for the school institution. In this paper we will analyze the guidelines defined in the existing Program of the Body Knowledge Area (Programa del Área del Conocimiento Corporal, ANEP, 2008), a programmatic document that intends to offer "a new look regarding the value of the game" To begin with, we took as a reference point the three axes the program introduces in connection with the game, i.e.: concept, methodology and content. In this articulation of categories, the program introduces a variety of goals and legitimations, evidencing some tensions. The main complexity of this research lies in addressing a double problem for physical education: the game perceived both as a pedagogical element and as an object of teaching. It is central to highlight that some of the problems identified in this analysis are not exclusive to this program, but reflect the ever-conflicting reality of the game in the
\end{abstract}


educational field in general and in physical education in particular.

Key words: game, physical education, school program.

\section{Resumo}

O jogo é um dos conteúdos fundantes da educação física escolar uruguaia. Sua inclusão como conteúdo a ser ensinado na escola data do primeiro programa de educação física escolar (1911). Desde essa inclusão temporã, podem rastrearse múltiplos discursos legitimadores. Em diferentes épocas foram-se estabelecendo diversas representações, imaginários e práticas em relação ao jogo. Propomos-nos visualizar os discursos atuais que dão sentido a este tradicional conteúdo. Optamos por focalizar a atenção no programa escolar vigente, entendendo que esse documento nos oferece uma possibilidade para rastrear aquilo que é definido e validado na actualidade para a instituição escolar. Neste texto analisamos os alineamentos que se definem no atual Programa do Área do Conhecimento Corporal (ANEP, 2008). Trata-se de um documento programático que pretende "uma nova mirada ao valor do jogo". Como ponto de partida, toma-se como referência os três eixos que apresenta o programa em torno do jogo, isto é: conceito, metodologia e conteúdo. Nesta articulação de categorias, o programa dá conta de diversos sentidos e formas de se-legitimar, evidenciando algumas tensões. A complexidade central desta pesquisa refere a um duplo problema para a educação física: o jogo como elemento pedagógico e o jogo como objeto de ensino. É importante assinalar que alguns dos problemas identificados nesta análise, não são exclusivos deste programa, sina que refletem uma realidade sempre conflictiva do jogo no âmbito educativo em general e na educação física em particular.

Palavras-chave: jogo, educação fisica, programa escolar. 


\section{Introducción.}

¿Es posible el juego en la escuela? Lo que denominamos como juego en la escuela, ¿es realmente juego?

Milstein y Mendes

El juego se presenta como un contenido de la educación física ${ }^{1}$ en Uruguay desde los primeros documentos oficiales. Será, junto con la gimnasia, lo que defina el espacio de la educación del cuerpo en la escuela desde finales del siglo XIX. Se puede observar su presencia en el primer documento oficial ${ }^{2}$ elaborado en 1911 y su permanencia en los diseños curriculares hasta la actualidad. Sin duda, en estos más de cien años, se pueden identificar diferentes sentidos y legitimaciones en tanto contenido de la EF escolar. Algunos de estos discursos continúan vigentes y coexisten con otros más actuales. Por ejemplo, en un principio, el juego dentro del ámbito educativo fue valorado por los beneficios fisiológicos que brindaba al niño. Así lo expresa Alejandro Lamas, nombrado en 1905, Profesor de Gimnasia de las escuelas de Montevideo:

La cultura física que realizamos en las escuelas [...] es incompleta; el ejercicio sistemado no es positivamente útil, llena solo una parte de esa cultura, [...] la que cumple condiciones pedagógicas de orden, disciplina y desarrollo intelectual de ciertas facultades, la que llena condiciones higiénicas, estéticas y condiciones correctivas de posibles deformidades, pero no la otra, la que pide mucho oxígeno para la sangre, mucha actividad para los pulmones, mucha sudoración para la piel, mucho grito para las cuerdas vocales, viva excitación para los centros nerviosos y mucha, muchísima alegría para el espíritu y mucho, muchísimo regocijo de vida. Esta segunda parte sólo pueden darla los juegos y ejercicios al aire libre (1912:4243)

\footnotetext{
1 En adelante EF

2 Denominado "Manual de ejercicios físicos" elaborado por Alejandro Lamas.
} 
Lejos de esta argumentación fisiológica, en la actualidad, pueden rastrearse distintas discursividades que pretenden rescatar el valor del juego como objeto cultural y asegurar esta práctica como un derecho del niño. Estos sentidos que la escuela, más precisamente la $\mathrm{EF}$, construye en relación al juego nos conducen a pensar en su pedagogización y en los rasgos particulares que asume esta práctica cultural cuando es introducida al interior de dicha institución. En ese instante, el juego, bajo la lógica escolar, suele posicionarse en el orden de lo útil y esto puede suponer algunas dificultades. Con respecto a esta complejidad, Nella (2009) sostiene que:

Desde un principio, el hecho de que un profesor, en la escuela, inicie cualquier situación de juego, se enfrenta con un gran límite que atenta contra lo que, por costumbre e históricamente, se llamó juego [...] Los niños siguen entonces ya no sólo las reglas internas propias del juego, sino la finalidad objetiva de la institución escolar y del profesor que conduce el juego (Nella, 2009: 231).

No estamos defendiendo una despedagogización del juego en la EF. Pretenderlo sería un error ya que todo "saber" que ingresa a la escuela necesariamente es tamizado por lo pedagógico. En todo caso, lo necesario e interesante es poder visualizar cómo el juego, en tanto objeto de enseñanza, es afectado por dicho proceso. Precisamente en este contenido hay una tensión particular ya que ha primado históricamente una concepción del juego más como una herramienta para enseñar otros contenidos o estimular y desarrollar ciertas capacidades que como un saber a ser enseñado. Intentaremos dar cuenta de estas y otras tensiones que pueden visualizarse en el programa escolar con respecto al juego.

\section{El Juego dentro del Programa}

Antes de introducirnos en el análisis de este contenido, nos parece necesario señalar de forma muy sintética algunas generalidades de este documento. Como rasgo principal diremos que este programa basa su fundamentación en marcos teóricos próximos a las ciencias sociales. Introduce una noción de sujeto, de cuerpo y de prácticas corporales que se aleja de los enfoques más tradicionales que han predominado en nuestro campo, es decir, las ciencias naturales. Construye una visión de EF donde el concepto de cultura corporal cobra singular importancia. Esto también se intenta reflejar en la fundamentación de cada 
contenido. El enfoque sobre el juego busca, por consiguiente, alinearse con este corrimiento teórico. De hecho se señala que:

En el transcurso del siglo XX en la Educación Física el juego fue perdiendo espacio al plantearse otras metas de rendimiento o al hacer énfasis en mejorar problemas posturales y musculares con un fuerte acento en la salud [...] En la actualidad se plantea una nueva mirada al valor del juego en la Educación Física Escolar (ANEP, 2008: 255)

Se señala que el juego "fue perdiendo espacio" en el ámbito de la EF por otras prioridades más vinculadas a la salud o al rendimiento. Sin embargo nos preguntamos, ¿existió una época donde el juego como contenido en sí mismo (y no el "juego-para”) tuvo centralidad en la EF escolar como para afirmar esta pérdida? También se advierte que este programa pretende una nueva "mirada" al valor del juego por lo que es factible visualizar nuevos discursos que legitimen su presencia en este programa. El desafío entonces es analizar este corrimiento teórico-conceptual con respecto al juego en el cual el programa intenta posicionarse.

Para comenzar, el juego es abordado desde tres enfoques o dimensiones que pueden resultar complementarios y que en su conjunto definen lo que es juego para este programa. Estos tres ejes se sintetizan en la siguiente definición que se explicita en el documento programático: "El juego en este programa se concibe como concepto, metodología y contenido. Es así que se convierte en contenido en la medida que es enseñable, y al mismo tiempo, en estrategia metodológica esencial para la enseñanza de otros contenidos.” (ANEP, 2008: 255). Por un lado se lo piensa como un concepto clave, que junto con corporeidad y motricidad proporciona un marco de referencia a la hora de fundamentar la EF como asignatura escolar. Por otro, forma parte de los saberes a ser enseñados al igual que el resto de los otros contenidos. Y finalmente se constituye como una herramienta metodológica fundamental para la enseñanza. Este enfoque plural del juego constituye un aspecto a problematizar en nuestro análisis. Algunas de estas preguntas podrían operar como disparadores: ¿qué concepto de juego se promueve?, ¿qué tensiones supone considerar que el juego es contenido y también estrategia metodológica?, ¿qué se pretende 
enseñar de este contenido? ¿Cuál es la naturaleza de este saber que se acondiciona para la enseñanza?

El camino que nos proponemos para ensayar algunas respuestas es el de analizar cada una de estas tres distinciones que el programa hace del juego.

\subsection{Juego como Concepto}

Creemos que los cuentos y los juegos son cosa de infancia. ¡Qué miopes somos! ¡Cómo podríamos vivir, en cualquier edad de la vida, sin cuentos y sin juegos!

Nietzsche

Si bien este eje o categoría es una de las tres dimensiones constitutivas del juego dentro del programa, cabe señalar que no se encuentran muchas referencias al respecto, constituyendo en sí una categoría un tanto invisible en este documento. En otras palabras, no aparece un encuadre teórico explícito de juego, sino más bien algunas ideas que intentan contextualizarlo. Con este propósito, rastreamos y citamos pasajes del programa que, a nuestro entender, dan pistas para interpretar cierto posicionamiento teórico-conceptual sobre esta práctica.

En el programa podemos leer: "El impulso lúdico, impulso natural e instintivo en el ser humano, debe ser tomado intencionalmente por el docente" (ANEP, 2008: 255). Esta frase si bien es muy breve, resulta de mucho interés ya que condensa una visión claramente muy presente en los discursos pedagógicos en torno al juego. Un primer aspecto a problematizar de este posicionamiento, refiere a la concepción de juego-natural. En varios sentidos, esta cita alude a una visión del juego como una cuestión natural, instalando así un primer debate. Pensar lo lúdico en términos de instinto o necesidad es un problema no menor para un documento que intenta arraigarse en lo cultural, más precisamente en la cultura corporal. En otras palabras, si concebimos que jugar es un acto instintivo, y podemos agregar fisiológico, tensiona en buena medida, la posibilidad de concebir el juego desde un enfoque histórico, cultural y político. En esta línea argumentativa, podríamos tropezar con otra idea muy presente en lo escolar ligada a la anterior, es decir, "los niños 
juegan por naturaleza", o "jugar está en su naturaleza". En este sentido, la propuesta de la EF en la escuela no sólo cumpliría una función desarrollista ante el instinto, sino que el compromiso es aún mayor, debería cuidarse de no reprimirlo. La escuela tiende a defender y propiciar el juego como una necesidad instintiva, un impulso natural, pero como tal tiene el cometido pedagógico de ordenarlo, marcando cuándo, cómo y a qué se juega.

El juego aparece como una actividad característica y exclusiva de lo que el niño hace. Coincidimos con Milstein y Mendes (1999), cuando plantean que el juego y la niñez aparecen como conceptos abstractos que dan cuenta de realidades universales. Es así que el niño por definición juega y el juego por definición también es propio del niño. En relación a la naturalización del juego, estos autores sostienen que:

La naturalidad del vínculo entre juego y niñez queda así establecida en términos biológicos o casi biológicos [..]. Esta naturalidad impide entender las transformaciones históricas que sufrieron las diversas maneras de existencia y de comprensión de la niñez y del juego [...]. Al no concebirlos como fenómenos sociales, estos significados "naturales" permanecen como fundamento no cuestionado en los debates educativos en torno al juego [...]" (Milstein y Mendes 1999: 62)

A la vez, esta lógica consolida otra falacia: el juego es propio de la infancia, y no del mundo adulto. Varios autores han señalado que la infancia es una categoría moderna ${ }^{3}, \mathrm{y}$ que las crías humanas anteriormente se educaban y socializaban participando directamente de las actividades adultas, incluidos los juegos.

Desde este discurso naturalizado nos introducimos ahora en una nueva categoría conceptual del juego que puede rastrearse en el programa: el juego-desarrollo. Muy emparentada con el discurso anterior aparece el juego en tanto herramienta de descubrimiento y crecimiento personal. El programa escolar plantea que "En el juego, acto social de dicho impulso, el individuo se muestra tal cual es, se expresa y se comunica con toda autenticidad". Continúa diciendo que el juego es un "[...] factor básico en el desarrollo

3 CFR Ariès 1960 
y en la construcción de la personalidad del niño" [...]. Debe contribuir al desarrollo de la corporeidad, de un sujeto creativo, expresivo, libre y reflexivo" (ANEP, 2008: 255). Se puede leer posteriormente una cita de Donald Winnicott, pediatra y psicoanalista inglés, en donde se afirma que es en el juego y sólo en él, donde el niño puede crear y usar toda la personalidad, el juego como espacio creador, permite al individuo descubrir su personalidad (Winnicot, cit. en ANEP 2008).

En esta categoría se despliega un discurso con aportes fundamentalmente biológicos y psicológicos, donde el juego permite el dominio de las diferentes etapas del desarrollo individual. El juego opera acompañando y estimulando el desarrollo motriz y cognitivo. Se justifica en el ámbito educativo ya que permite desarrollar ciertas capacidades y esquemas en el niño. Este enfoque desarrollista ha estado muy ligado a los sentidos y legitimaciones de la EF en la escuela, operando de forma muy solidaria con este propósito. Puede resultar interesante rastrear las posibles alianzas de este discurso sobre el juego (en el sentido desarrollista) con la creciente psicologización de la educación, el advenimiento del constructivismo, intensificadas con los aportes de la corriente psicomotriz de la EF consolidada en la década de los 90 .

A estas dos categorías, se suma un tercer discurso que da sentido al juego en el programa. Nos referimos a una categoría más actual, producto de políticas sociales y educativas propias de esta época, es decir, el juego como derecho. Desde el programa se argumenta:

La Educación Física en la escuela se fundamenta, además, en los Artículos 28, 29 y 31 de la Declaración de los Derechos del Niño (1989). Se hace mención al derecho de los mismos a la educación para desarrollar sus capacidades mentales y físicas. Esto incluye el derecho al juego, al buen uso del tiempo libre y a las actividades recreativas [...] (ANEP, 2008, 236).

Desde este discurso jurídico se concibe el juego como un derecho del niño, siendo la educación su garante. Afirmado como derecho, la sociedad y el estado tienen responsabilidades frente al juego y deberán velar por su cumplimiento. Una vez más es 
necesario detenernos en este nuevo discurso que se promueve desde el programa e intentar visualizar las complejidades que en términos operativos y simbólicos conlleva.

Al interior de la institución escolar el derecho al juego puede verse truncado ya que la infraestructura, los recursos y condiciones para su cumplimiento no son siempre favorables. Víctor Pavía, referente del juego a nivel regional, quien ha problematizado esta cuestión se pregunta: ¿cómo forma la escuela primaria de hoy para el ejercicio pleno del derecho al juego de todos los niños y niñas, en espacios compartidos, seguros, amplios y confortables? (Pavía, 2001). Sin dudas esta preocupación tiene validez para las escuelas públicas uruguayas en las cuales los recursos materiales, así como los espacios y tiempos de trabajo no son siempre óptimos. En estas circunstancias desfavorables, velar por el derecho al juego, se transforma en todo un desafío para los docentes.

A estos obstáculos instrumentales y operativos podríamos agregar otros de carácter simbólico vinculados a la función específica de la escuela, preparar para el mundo adulto perpetuando códigos sociales de clase (Varela y Álvarez Uría 1991). En este sentido la escuela, en su intención de educar a la clase trabajadora según los intereses burgueses, establece una temporalidad donde el tiempo libre y de ocio está condicionado por el tiempo de trabajo. El derecho al juego en la escuela, tiende a estar determinado por el tiempo de rendimiento, de organización y de gobierno de la infancia que caracteriza al proceso de escolarización.

\subsection{Juego como Metodología}

El juego como mandato no es juego, todo lo más, una réplica, por encargo, de un juego.

Huizinga

Para este programa, como ya fue señalado, el juego también es valorado en tanto que permite la enseñanza de otros contenidos. En el programa se señala que "El juego por su carácter integral implícito en la actividad lúdica debe atravesar el período escolar con un rol central. Se constituye en una excelente estrategia metodológica para la enseñanza de otros contenidos" (ANEP, 2008: 260). Este juego-metodología que establece el programa, por supuesto no es ninguna novedad en el ámbito educativo y menos puede considerarse como 
una estrategia exclusiva de la EF. El binomio juego-educación en la modernidad, puede ser rastreado, entre otros, en la pedagogía romántica de Froebel. Según Stagno:

[...] Froebel, propuso la denominación "jardín de infantes", en relación con la posibilidad que tendrían los niños para crecer, desarrollar y madurar en forma natural (...). El juego, creado por y para los niños fuera de la institución escolar, era revestido de un fin educativo, se lo transformaba en un medio de enseñanza (Stagno, 2011:53)

En la actualidad, este enfoque del juego se ha expandido y consolidado como una verdad absoluta. El juego en aquel Kindergarten creado por Froebel en 1840, continúa, sin temor a equivocarnos, en plena vigencia. Pero especialmente en el campo de la EF, somos más froebelianos que ningún otro. En primer lugar, la utilización masiva de "juegos" (si es que se pueden llamar juegos) como recurso metodológico casi exclusivo, es de por sí cuestionable. Desde esta visión se espera que la enseñanza (y el aprendizaje) del deporte, la gimnasia, las actividades expresivas, las actividades acuáticas, entre otros contenidos del programa, sea posible gracias al juego. Surgen así los juegos de, juegos para, juegos con, juegos y más juegos que nos facilitarán estos procesos. El juego, piedra filosofal de nuestra profesión, se convierte en la herramienta adecuada para todo tipo de contenidos ya que el juego vale para todo y todo se soluciona con un juego (López Pastor, 2004). Siguiendo a este autor, lo lúdico parece ser el símbolo de progreso, innovación y profesionalidad en EF. La diferencia entre los antiguos profesores y los actuales consiste fundamentalmente en la aplicación o no del juego para la enseñanza de los contenidos que por otra parte, no se cuestionan demasiado (op cit, 2004).

En segundo lugar, concebir el juego como herramienta metodológica nos conduce a una compleja discusión sobre la estructura y lógica propia del juego. En este sentido, y siguiendo la distinción que hace Pavía entre "formas y modos" de jugar, el juego como medio de enseñanza, ya no supone un “juego transparente”, juego en sí, más bien refiere a un "juego paradójico" donde la invitación a jugar tiene forma de juego pero el modo de 
jugar no es lúdico. ${ }^{4} \mathrm{Si}$ les decimos "vamos a jugar" a nuestros alumnos y el fin es enseñar una técnica deportiva ¿es realmente un juego? En este caso, estaríamos en presencia de un juego amenazado, donde jugar por mandato es no jugar, es un falso orden (Scheines, 1993). Esta autora nos advierte: "El juego no puede reducirse a un recurso didáctico más. No es cuestión de remplazar 'la letra con sangre entra' por 'la letra con juego entra' como si se cambiaran figuritas o se sustituyera una tecnología por otra”. (Scheines, 1998:59).

Jugar con este fin supone, según Milstein y Mendes (1999), transformar al juego en una actividad educativa que produce una práctica totalmente distinta a los juegos que las sociedades han producido y reproducido históricamente.

Concebido como una práctica social, el juego y sus diversas formas de articularse en la vida cotidiana, puede entenderse como un conjunto de modos de reproducir relaciones y significados en un plano específico, cuya particularidad reside en permitirle a los sujetos salir de un plano, dimensión o aspecto de la realidad para ingresar en una dimensión ficcionalizada de la misma [...] El juego, transformado en una actividad educativa bajo la regulación de la lógica escolar, poco tiene que ver con el juego como práctica social en los términos antes planteados (1999: $68,69)$

Como podemos observar, el juego como metodología posibilita distintos ángulos de análisis sobre lo que conocemos como juego y sus transformaciones en la práctica educativa.

\subsection{Juego como Contenido}

¿Qué enseñamos del jugar los docentes de Educación Física?

V. Pavía

En esta última dimensión de análisis, abordaremos el planteo del programa en relación al juego como contenido, que ya hemos adelantado. En este punto, recordamos que para este

\footnotetext{
${ }^{4}$ La forma refiere a la configuración general de un juego, su apariencia externa, es lo que nos permite diferenciar un juego de otro (sentido, lógica interna, fuente de emoción, tenor de las acciones). El modo, sin embargo, da cuenta de la participación en el juego, es decir, si quien participa lo vive como tal o no. Es de índole más cualitativo, implica entre otras cosas, fingimiento auténtico, permiso, confianza, empatía, complicidad (Pavía, 2010).
} 
documento "se convierte en contenido en la medida que es enseñable [...]". (2008:255). Se imponen de inmediato una serie de preguntas de diversa índole: ¿qué es lo enseñable del juego para el programa?, o siguiendo a Pavía (2010), ¿qué del jugar termina siendo un contenido curricular en EF? Por otro lado, ¿Saber y contenido son lo mismo? ¿La calidad de "contenido" es lo que lo convierte en enseñable o su constitución como un saber de la EF?

Dado que entendemos que algo es enseñable a partir de que existe un saber/conocimiento que es necesario poner en signos, proponemos invertir el proceso a partir de las preguntas: ¿existe algo parecido a un saber del juego en la EF? En la creación de conocimiento sobre el juego como parte de la herencia cultural de humanidad (en términos de cultura corporal), ¿hay algo que necesite ser puesto en signos para las nuevas generaciones? ¿Qué dice o puede decir de él la EF? ¿Cómo transponer este saber de forma tal que no pierda la esencia de su existencia como saber? Si todas estas preguntas tienen cierta dimensión de posibilidad, entonces será necesaria su transposición y devendrá contenido en la dimensión política de la enseñanza como parte del curriculum.

Si bien el análisis realizado hasta el momento no permite objetivar un saber del juego en tanto objeto teórico, buscaremos avanzar en este problema a partir del análisis de los saberes designados para la enseñanza del juego. Para una mejor comprensión del esquema, es necesario señalar que el programa prescribe los contenidos en cuatro niveles. Se distinguen entonces, el nivel inicial que abarca la etapa preescolar (3 a 5 años), el primer nivel en el cual se incluye a 1er y 2do grado (6 a 8 años), el segundo nivel lo constituye 3er y 4to grado ( 8 a 10 años) y por último, 5to y 6to grado (10 a 12 años) se ubican en el tercero completando así el ciclo escolar. El contenido juego tiene la particularidad de estar presente en todos los niveles. 


\begin{tabular}{|c|c|}
\hline \multicolumn{2}{|r|}{ Juego como contenido - ANEP - Programa $2008^{5}-$} \\
\hline Juego Simbólico & $\begin{array}{l}\text { - Si bien el juego simbólico está presente en todo el ciclo escolar, se presentan } \\
\text { más orientaciones y modalidades para los primeros niveles dando cuenta de una } \\
\text { mayor presencia de este tipo de juego en los primeros años de escolarización. } \\
\text { - Se sugieren los juegos de roles e imitación con pocos personajes y reglas } \\
\text { simples para el nivel inicial. También se proponen los juegos libres (no se hace } \\
\text { ninguna mención al juego libre). } \\
\text { Para el primer nivel se continúa con los juegos de roles con aumento de } \\
\text { personajes que pueden ser permanentes o cambiantes y reglas más complejas. } \\
\text { También aparecen los juegos de fantasía, los espacios cambiantes y } \\
\text { estructurados (refugios). } \\
\text { Para el segundo y tercer nivel se sugieren juegos simbólicos relacionados con } \\
\text { otros contenidos (sólo se menciona este comentario). }\end{array}$ \\
\hline Juegos Reglados & $\begin{array}{l}\text { - En este caso también se observa una secuenciación del juego reglado desde el } \\
\text { nivel inicial hasta el tercer nivel escolar (5to y 6to grado). } \\
\text { - Los juegos de persecución y con consignas constituyen la modalidad central en } \\
\text { este contenido. La diferencia para los distintos niveles radica en la cantidad y } \\
\text { complejidad de las reglas. Se proponen pocas y simples para el nivel inicial y a } \\
\text { medida que se avanza en los niveles aumenta la variedad y complejidad de las } \\
\text { mismas. } \\
\text { Para el segundo nivel se proponen juegos de persecución con la posibilidad de } \\
\text { crear reglas por parte de los alumnos y en escenarios variables. También se } \\
\text { incorporan los juegos de colaboración y oposición. } \\
\text { En el tercer nivel, están presentes únicamente los juegos de colaboración y } \\
\text { oposición con aumento en la cantidad y complejidad de reglas. Se propone la } \\
\text { creación y modificación de los juegos. }\end{array}$ \\
\hline $\begin{array}{c}\text { Juegos } \\
\text { cooperativos }\end{array}$ & $\begin{array}{l}\text { - Este contenido no está presente en el nivel inicial. Se prescribe a partir del } \\
\text { primer nivel. La lógica que predomina en las orientaciones refiere a la cantidad } \\
\text { de participantes y en la resolución del problema que se plantea en el juego } \\
\text { cooperativo. } \\
\text { - De esta forma, se pasa de grupos pequeños para el primer nivel, a grandes } \\
\text { grupos o el grupo en su totalidad para el segundo y tercer nivel. } \\
\text { Lo mismo ocurre con la resolución al desafío planteado por el juego } \\
\text { cooperativo. Se sugiere resoluciones básicas de situaciones problema en } \\
\text { primer nivel, a estrategias de resolución social para los otros dos niveles. }\end{array}$ \\
\hline $\begin{array}{c}\text { Juegos } \\
\text { tradicionales }\end{array}$ & $\begin{array}{l}\text { - } \quad \text { Este es el único caso en el que el programa no hace distinción por niveles. } \\
\text { - Se presenta como un contenido para trabajar durante todo el ciclo escolar. } \\
\text { - Se sugieren juegos en ronda, en dispersión, con cancha dividida, en } \\
\text { diferentes espacios físicos, cantados, con palmas y con elementos. }\end{array}$ \\
\hline
\end{tabular}

Lo primero que nos interesa señalar es que en el programa, cuando se intenta explicitar el juego como contenido, se opta por distinguir cuatro modalidades o categorías de juegos que deberían ser enseñados, ellos son: simbólicos, reglados, cooperativos y tradicionales. Es difícil encontrar un criterio orientador de esta taxonomía, lo que nos

${ }^{5}$ Cuadro de elaboración propia en base a lo que se explicita en el programa. Los textos en negrita son citas textuales del programa. 
sugiere que es un listado producto de recortes de diferentes clasificaciones. Los juegos “reglados" y "simbólicos" parecen referir al criterio de juegos piagetiano", sin que esté presente en el programa el juego ejercicio como tercer elemento del sistema del autor.

En cuanto al juego cooperativo puede resultar interesante preguntarse por la argumentación y el sentido de estos juegos. ¿Constituye un intento de tomar distancia de la competencia? ¿Qué es un juego cooperativo? ¿A qué categorización responde? El programa no muestra muchas pistas para responder estas preguntas. No obstante, entendemos que su inclusión puede relacionarse a una preocupación pedagógica por los modos vinculares, más cercana a la educación en valores que a la enseñanza de un saber de la EF. En este caso, tampoco aparecen otros elementos del mismo criterio taxonómico como los juegos que priorizan las relaciones de oposición y de colaboración-oposición.

La incorporación de los juegos tradicionales podría justificarse como un saber en sí mismo, como planteamos anteriormente en este trabajo, pero la justificación de los mismos no tiene un sentido cultural (juegos locales, regionales, internacionales, propios de otras culturas, rastreo histórico de juegos, entre otros) sino por criterios organizativos (en ronda, diferentes espacios físicos, por ejemplo).

Otro aspecto que nos parece relevante demarcar es que algunos juegos se prescriben desde el nivel inicial, y otros, aparecen en los niveles superiores. Es decir, la temporización y secuenciación responden a una lógica que podríamos identificar con los principios célebres: "de lo fácil a lo difícil", "de lo sencillo a lo complejo"7 . Atendiendo a esta lógica, el juego como contenido significa, justamente, enseñar una variedad de juegos, en los cuales prevalecerá el juego libre ${ }^{8}$, la fantasía, y consignas simples para los primeros niveles, y juegos más complejos en relación a las reglas y estructura en los niveles superiores. Este planteo, si no es apoyado por otras orientaciones y/o saberes, puede presentar algunas debilidades para un programa que intenta fundamentarse desde el

\footnotetext{
${ }^{6}$ Según palabras de Jean Piaget existen "tres grandes tipos de estructuras que caracterizan los juegos infantiles y dominan la clasificación de detalle: el ejercicio, el símbolo y la regla; ( Piaget, 1961, p.153)

7 Principios que pueden rastrearse en el pedagogo alemán Wolfang Ratke (siglo XVII), sin embargo, alcanzaron mayor difusión en la obra de Comenio.

${ }^{8}$ Es complejo interpretar a qué tipo de juego se está refiriendo el programa con "juego libre".
} 
concepto de cultura corporal. Desde el inicio señalamos el intento de cambio de este programa en relación al distanciamiento de marcos teóricos que tradicionalmente dieron sustento a nuestro campo disciplinar (biología, fisiología, psicología). Este corrimiento supone por tanto, pensar el juego como una práctica corporal contextualizada, en la que se evidencian aspectos culturales, históricos, éticos y políticos. ${ }^{9}$. En este sentido, coincidimos con Vaz (2015) cuando afirma:

Los juegos son formas de expresión cultural de los diversos pueblos. Un juego expresa, como acontece con otros elementos de la cultura, un conjunto de concepciones, valores y normas que se distinguen por el tiempo en que surgen y son practicados. Es probable que todas las sociedades posean actividades lúdicas organizadas en forma de juegos, lo cual les atribuye cierto carácter de universalidad, mostrando que son una de las formas de organización más constantes de la condición humana. (Fernández Vaz 2015: 129).

Al momento de explicitar aquello que debería enseñarse en relación al juego, el programa no logra plasmar este cambio teórico-conceptual, se limita a presentar únicamente modalidades de juegos organizados y prescriptos según edades y niveles escolares.

\section{Consideraciones finales}

En el transcurso de este trabajo intentamos reflexionar en torno al juego y la EF, tomando como punto de partida el programa escolar. Como ya fue señalado, dicho documento propone abordarlo como concepto, metodología y contenido, intentando un nuevo posicionamiento del juego en la EF escolar. El propósito de este escrito consistió en rastrear y analizar los discursos que subyacen en cada una de estas dimensiones para evidenciar algunas tensiones. Es importante señalar que algunos de los problemas identificados en este análisis, no son exclusivos de este programa, sino que reflejan una realidad siempre conflictiva del juego en el ámbito escolar y en EF. Tal como nos dice Pavía:

\footnotetext{
9 Para una distinción entre actividades y prácticas corporales se recomienda el artículo de Crisorio (2015).
} 
[...] el jugar en la escuela presenta formas y modos tan variados que no siempre que alguien dice ¡Vamos a Jugar! está hablando exactamente de la misma cosa; un hecho que por sí mismo ya justifica la construcción de precisiones que permitan acuerdos mínimos, tanto a nivel de las prescripciones curriculares como de los mandatos metodológicos (2010: 91).

En el análisis del juego en este documento, pudimos observar que tanto a nivel conceptual, como en su perspectiva metodológica hay aspectos que necesitan ser revisados para desnaturalizar discursos que condicionan directamente la enseñanza de este contenido. Es así que el juego en el programa se convierte en un pilar de la EF escolar, colabora con los objetivos generales de la educación primaria, es una herramienta clave para la enseñanza, a la vez que se declara como un contenido enseñable. Es necesario examinar algunas de estas "precisiones" para lograr, como propone el programa, una nueva mirada al valor del juego en la EF escolar. Entendemos que esta pretensión es muy valiosa pero presenta ciertas debilidades y no termina por constituir acontecimiento, fundamentalmente en la concepción y en el tratamiento que se hace del juego como contenido.

Proponemos distinguir las necesidades didácticas de las implicancias conceptuales. Nos referimos a la simplificación implícita en el afirmar que el juego es contenido a partir de que es enseñable, algo especialmente significativo. Entendemos, de acuerdo a nuestro marco de referencia teórico, que la naturaleza del saber afecta a la enseñanza (Behares 2011). Lo que el programa propone al dividir entre concepto y contenido transforma al juego en un producto tecnológico, en tanto su esencia es servir a la tecnología de la enseñanza.

La idea del juego como concepto planteada en el programa no tiene un resorte en su conceptualización como saber. Aparece como necesidad, como factor de desarrollo y como derecho, pero no como una noción o conceptualización que identifique la naturaleza del saber del juego, ni su construcción histórica como práctica corporal. 
Entendemos que la presencia del juego como contenido de la EF supone la investigación de estas formas lúdicas surgidas en contextos y tiempos determinados (Fernández Vaz 2015) y su trasmisión a las nuevas generaciones como capital cultural ${ }^{10}$. Esto no sólo identifica la trasmisión de ciertos juegos, generalmente identificados como juegos tradicionales de ciertas comunidades, sino la investigación del juego como objeto teórico y problema epistemológico de la EF, su sentido como práctica corporal, sus relaciones con el tiempo de trabajo y de ocio y sus vínculos y diferencias con otros saberes de la EF. En este sentido consideramos que "el juego" en el programa se vacía de significado, para dar lugar a distintos juegos o a "los juegos", donde importa más la cantidad de juegos que el sentido del juego en la escuela, y su trasmisión en tanto capital corporal (Barbero, 2007).

En relación al juego como medio, sea en el sentido de favorecer el desarrollo, canalizar necesidades o como metodología, entendemos que es necesario mantener una actitud alerta para no confundirnos. El juego de por sí ya presenta la suficiente complejidad para ser enseñado en la escuela por su carácter inútil, como para someterlo a la dinámica de ser útil para. Parece ser necesario circunscribir a lo metodológico por un lado y al juego como saber por otro.

El jugar como parte del curriculum supone una teoría del juego y no la mecánica didactista de su implementación en la clase de EF. En este marco el enseñante, en tanto posición privilegiada en relación al saber, no dispone de un objeto concreto a ser traspuesto sino de una teoría, de un saber sobre el juego del que es portador frente a un otro. Ese saber puede ser tanto un juego (por ejemplo un juego tradicional) como el habilitar un lugar para ser apropiado por el niño, donde el encuentro con el otro sea posible de modo lúdico. Esto no significa "pelota al medio" ni "juego libre", sino enseñanza de un saber complejo de poner en signos sin desfigurarlo en el proceso.

Si bien este documento muestra diferencias sustanciales con respecto a programas escolares anteriores, el juego sigue sosteniéndose desde una perspectiva desarrollista. Este enfoque

\footnotetext{
${ }^{10}$ En el sentido acuñado por Pierre Bourdieu (1988)
} 
psicologizante y biologicista representa una seria dificultad para tematizar y enunciar contenidos desde una visión curricular que propone aproximarse al concepto de "cultura corporal". Como ejemplo de esta situación podemos mencionar que en el rastreo realizado en el programa en busca de elementos conceptuales, no visualizamos una categoría que diera cuenta del juego como juego-objeto cultural.

No pretendemos agotar la discusión, por el contrario sería deseable que este trabajo fuera también interpelado por colegas. Se trata de eso, cuestionar y problematizar junto a otros las discursividades que han operado históricamente como verdades en los diferentes contenidos de la EF escolar, y que sin duda han determinado y determinan ciertas prácticas de enseñanza. Es ahí donde pretendemos colaborar con este diálogo.

\section{Referencias bibliográficas}

Administración Nacional de Educación Pública (ANEP) (2008). Programa de Educación Inicial y Primaria. Consejo de Educación Inicial y Primaria, Montevideo.

Aries. P. (1960) L'enfant et la vie familliale sous l'Ancien régime. Plon.

Barbero, J. I. (2007) Capital(es) corporal(es) que configuran las corrientes y/o contenidos de la educación física escolar. En Ágora para la Educación Física y el Deporte, 4-5, 21-38.

Behares L. E. (2011) Enseñanza y producción de conocimiento. La noción de enseñanza en las politicas universitarias uruguayas. Montevideo: Departamento de Publicaciones de la Universidad de la República.

Bourdieu, P. (1988). Cosas dichas. Buenos Aires: Gedisa.

Crisorio, R. (2015), Actividad(es) Física(s) vs Practica(s) Corporal(es) en, Cuerpo, Educación, Política. Tensiones epistémicas, históricas y prácticas. En Galak, E. y Gambarotta, E. (edit), Cuerpo, educación y politica. Tensiones epistémicas, históricas y prácticas. Buenos Aires: Biblos.

Fernández Vaz, A. (2015) Juegos y deportes: desafíos para la Educación Física. En Galak, E. y Gambarotta, E. (edit), Cuerpo, educación y política. Tensiones epistémicas, 
históricas y prácticas. Buenos Aires: Biblos.

Lamas, A. (1912) Educación física é intelectual conexas. Un plan de educación física infantil. Montevideo: Barreiro y Ramos.

. (1911) Manual de ejercicios físicos para las escuelas públicas. Montevideo: Barreiro y Ramos.

López Pastor, V. (2004) Una mirada crítica sobre las prácticas pedagógicas en educación física. En revista Novedades Educativas, Año 16, nº 157, 4-5.

Milstein, D. y Mendes, H. (1999) La escuela en el cuerpo. Madrid: Miño y Dávila Editores.

Nella, J. (2009) El juego: un contenido inútil. En Crisorio. R y Giles, M. Estudios críticos de Educación Física. La Plata: Ediciones Al margen.

Pavía, V. (coord.) (2010) Formas del juego y modos de jugar. Secuencias de actividades lúdicas. Neuquén: Editorial Universitaria, Universidad Nacional del Comahue.

. (2001) Un espacio para el juego, en revista digital www.efdeportes.com, Año 7, $\mathrm{N}^{\mathrm{o}} 41$, octubre de 2001, Buenos Aires

Piaget, J. (1961) La formación del símbolo en el niño. Imitación, juego y sueño. Imagen y representación. México: Fondo de cultura económica.

Scheines, G. (1998) Juegos inocentes, juegos terribles. Buenos Aires: Editorial Universitaria de Bs As.

- (1993) Problemas del juego en la Educación Física. ler Congreso de Educación Física y Ciencias, FaHCE-UNLP, 1993, La Plata

Stagno, L. (2011) El descubrimiento de la infancia, un proceso que aún continúa. En Finocchio, S y Romero, N (comp) Saberes y prácticas escolares. Rosario: Homo Sapiens Ediciones.

Varela. J. y Alvarez Uria, F. (1991) La arqueología en la escuela. Madrid: Las Ediciones de La Piqueta. 